\title{
Westfall Young Adjustment
}

National Cancer Institute

\section{Source}

National Cancer Institute. Westfall Young Adjustment. NCI Thesaurus. Code C72514.

An adjustment method used in multiple comparison procedures to calculate an adjusted probability. This method ranks probabilities in descending order of their absolute p-values first, then calculate the adjusted p-values by an algorithm described in Dudoit et al. 2003. 